Available online at www.sciencedirect.com

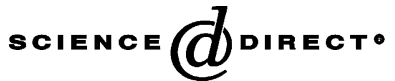

Applied Catalysis A: General 245 (2003) 289-301

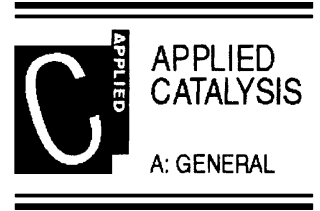

www.elsevier.com/locate/apcata

\title{
Diffusion and chemical reaction in isoamylene etherification within a cation-exchange resin
}

\author{
Päivi K. Pääkkönen*, A. Outi I. Krause \\ Laboratory of Industrial Chemistry, Helsinki University of Technology, P.O. Box 6100, 02015 Hut, Finland
}

Received 26 August 2002; received in revised form 30 November 2002; accepted 4 December 2002

\begin{abstract}
The effects of temperature (323-353 K) and the molar ratio of the reagents methanol/isoamylenes $(0.3-3.0)$ on the formation rates of tert-amyl methyl ether (TAME) were measured with different Amberlyst 16 (A16) particle sizes in a continuous stirred tank reactor. The effect of catalyst swelling was studied with several mixtures of reagents (MeOH and IA) and product (TAME) on Amberlyst 16. The particle size influenced the steady state reaction rates most notably at higher temperatures and with substoichiometric feed of the reagents (methanol/isoamylenes), i.e. when the reaction rate should be fast. The estimated effectiveness factors $(0.5-1.0)$ decreased with increasing particle size and increasing temperature. At nonstoichiometric feed ratios of the reagents the value of the estimated effectiveness factor decreased more when methanol was fed in excess. Recalculations of the earlier results of TAME-synthesis demonstrated that when modifying the rate constants obtained from the batch reactor experiments by dividing them by the effectiveness factors, the R-squared values of the regression analysis against temperature increased (improved fit for Arrhenius-type dependency) and the activation energies increased by about $9 \mathrm{~kJ} \mathrm{~mol}^{-1}$. (c) 2002 Elsevier Science B.V. All rights reserved.
\end{abstract}

Keywords: Cationic ion-exchange resin; tert-Amyl methyl ether (TAME); Isoamylenes; Intraparticle diffusion; Reaction kinetics

\section{Introduction}

The reformulated gasoline component TAME (tert-amyl methyl ether, 2-methoxy-2-methylbutane) is commercially manufactured in liquid phase using strongly acidic macroporous ion-exchange resins as the catalyst. Conventional ion-exchange resins are copolymers of divinylbenzene (DVB) and styrene, sulfonic acid being the active site (Brønsted acidity). Lewis acidity (free electron pairs of oxygen) has also been proposed [1]. The amount of divinylbenzene can vary from 12 to $20 \mathrm{wt} . \%$, and it directly determines

\footnotetext{
* Corresponding author. Tel.: +358-9-4512666; fax: +358-9-4512622.

E-mail address: paakkonen@polte.hut.fi (P.K. Pääkkönen).
}

the degree of crosslinking and the rigidness of the structure, i.e. the more DVB in the catalyst, the more rigid the resin and its macroporous structure. Resin catalysts have, however, a bidisperse structure with a microporous gel phase also present. The resins swell in polar solvents: those with less DVB swell more than the ones with more DVB. The number of sulfonic acid groups can typically vary from $4.8 \mathrm{mmol} \mathrm{g}^{-1}$ (e.g. conventionally sulfonated Amberlyst 15) to $5.2 \mathrm{mmol} \mathrm{g}^{-1}$ (e.g. hypersulfonated Amberlyst 35). In addition to sulfonic acid content, the catalytic activity of the resins is determined by the polarity of the reaction medium and also by resin structure [2].

Several authors have studied the intraparticle masstransfer effects with ion-exchange resins in various reactions. Gupta and Douglas [3] determined the 


\begin{tabular}{|c|c|}
\hline \multicolumn{2}{|c|}{ Nomenclature } \\
\hline$a_{i}$ & activity of a component $i=\gamma_{i} x_{i}$ \\
\hline$C$ & concentration $\left(\mathrm{mol} \mathrm{m}^{-3}\right)$ \\
\hline$D$ & $\begin{array}{l}\text { molecular diffusion (bulk diffusion) } \\
\text { coefficient }\left(\mathrm{cm}^{2} \mathrm{~s}^{-1}\right)\end{array}$ \\
\hline$D_{\mathrm{e}}$ & $\begin{array}{l}\text { effective diffusion coefficient } \\
\left(\mathrm{cm}^{2} \mathrm{~s}^{-1}\right)\end{array}$ \\
\hline$E_{\mathrm{a}}$ & activation energy $\left(\mathrm{J} \mathrm{mol}^{-1}\right)$ \\
\hline$F_{i}$ & molar flow of component $i\left(\mathrm{~mol} \mathrm{~s}^{-1}\right)$ \\
\hline$\left[\mathrm{H}^{+}\right]$ & $\begin{array}{l}\text { acid capacity of the catalyst } \\
\left(\mathrm{mol} \mathrm{kg}^{-1}\right)\end{array}$ \\
\hline$k$ & rate constant $\left(\mathrm{mol} \mathrm{kg}^{-1} \mathrm{~s}^{-1}\right.$ or $\left.\mathrm{s}^{-1}\right)$ \\
\hline$K_{i}$ & $\begin{array}{l}\text { adsorption equilibrium constant for } \\
\text { component } i\end{array}$ \\
\hline$K_{j}$ & $\begin{array}{l}\text { reaction equilibrium constant for } \\
\text { reaction } j, j=1-3\end{array}$ \\
\hline$\dot{m}_{\text {tot }}$ & total flow $\left(\mathrm{kg} \mathrm{s}^{-1}\right)$ \\
\hline$M_{i}$ & molar mass of component $i$ \\
\hline$r_{i}$ & $\begin{array}{l}\text { rate of reaction for component } i \\
\left.\left(\mathrm{~mol} \mathrm{~kg}^{-1} \mathrm{~s}^{-1} \text { or s}\right)^{-1}\right)\end{array}$ \\
\hline$R$ & radius of the particle $(\mathrm{mm})$ \\
\hline$R^{2}$ & $\begin{aligned} \text { regression }= & 1-\sum_{n}\left(y_{\mathrm{exp}}-y_{\mathrm{est}}\right)^{2} / \\
& \sum_{n}\left(y_{\exp }-\bar{y}\right)^{2}\end{aligned}$ \\
\hline$s$ & under surface conditions \\
\hline$T$ & temperature $(\mathrm{K})$ \\
\hline$V$ & $\begin{array}{l}\text { molar volume of the component } \\
\left(\mathrm{cm}^{3} \mathrm{~mol}^{-1}\right)\end{array}$ \\
\hline$W_{\text {cat }}$ & catalyst mass $(\mathrm{kg})$ \\
\hline$w_{i}$ & weight fraction of component $i$ \\
\hline$x_{i}$ & molar fraction of component $i$ \\
\hline \multicolumn{2}{|c|}{ Abbreviations } \\
\hline 2M1B & 2-methyl-1-butene \\
\hline $2 \mathrm{M} 2 \mathrm{~B}$ & 2-methyl-2-butene \\
\hline A16 & Amberlyst 16 (Rohm\&Haas) \\
\hline DVB & divinylbenzene \\
\hline IA & $\begin{array}{l}\text { isoamylenes mixture } \\
\text { (93 wt.\% 2M2B, } 7 \text { wt.\% 2M1B) }\end{array}$ \\
\hline $\mathrm{MeOH}$ & methanol \\
\hline TAME & $\begin{array}{l}\text { tert-amyl methyl ether, } \\
\text { 2-methoxy-2-methylbutane }\end{array}$ \\
\hline \multicolumn{2}{|c|}{ Greek letters } \\
\hline$\gamma_{i}$ & activity coefficient for component $i$ \\
\hline$\varepsilon$ & porosity \\
\hline
\end{tabular}

\begin{tabular}{|ll|}
\hline$\eta$ & effectiveness factor \\
$\mu$ & viscosity (Pa s) \\
$\rho_{\mathrm{p}}$ & density of a catalyst particle dry/swollen \\
& $\left(\mathrm{kg} \mathrm{dm}^{-3}\right)$ \\
$\tau$ & tortuosity \\
$\phi$ & Thiele modulus \\
$\Phi$ & Weisz-Prater criterion \\
\hline
\end{tabular}

chemical reaction and diffusion rate parameters for the hydration of liquid isobutylene to tert-butanol with a gellular Dowex $50 \mathrm{~W}$ (8\% DVB) as the catalyst. High reaction rates were found to result from a high diffusivity of isobutylene within the resin catalyst. Both the high value for diffusivity and its negative temperature coefficient were interpreted to be evidence of the transport mechanism within the resin, this being the surface diffusion of isobutylene in an adsorbed state. Velo et al. [4] studied the hydration of isobutylene with Amberlyst 15 (DVB 20\%). Intraparticle diffusivity was found to increase with temperature and decrease with increasing tert-butanol concentration. Apparent values for the tortuosity factor changed from 1.3 (at high temperature in pure water) to about 4.5 (at low temperature in TBA-rich solutions). Their calculations were based on the deviations of the group $D_{\mathrm{e}} \mu$ from the Stokes-Einstein equation relating solvent viscosity and molecular diffusion $(D \mu / T=$ constant). Ihm et al. [5] used two different types of macroreticular resin catalysts in the hydration of isobutylene: Amberlyst XN-1010 (DVB 85\%) and Amberlyst 15. The diffusion-limited reaction was interpreted with a two-phase model in which the internal active sites (inside gel microspheres) were assumed to be more active than the external ones (those on the gel microparticles). Berg and Harris [6] considered the general problem of accounting for multicomponent diffusion effects in liquid-filled heterogeneous catalysts and applied the procedure for MTBE-synthesis. They described the molecular diffusion processes within the catalyst using generalised Maxwell-Stefan (GMS) equations. The resulting species conservation equations were reduced to a single ordinary differential equation through applying invariant solutions, i.e. assuming that the relative changes in the molar fractions of the reacting species are linearly related for a constant product of liquid 
molar density and of a matrix of modified Fick diffusion coefficients. GMS equations taking the chemical potential gradients as real driving forces were also applied to MTBE-synthesis by Sundmacher and Hoffmann [7]. Recently, a dusty-fluid model (DFM) was applied to the synthesis of ETBE [8] and of MTBE and TAME [9]. DFM incorporates ordinary diffusion and matrix diffusion (Knudsen for gases) accounting for friction between species and porous medium, as well as convection. Pla et al. [10] estimated effectiveness factors theoretically for the synthesis of MTBE in the liquid-phase. At the usual operation conditions in industrial reactors, effectiveness factor ranging from 0.7 to 1.0 were found. In this study, we present the results of TAME-synthesis with different particle size fractions of Amberlyst 16 as the catalyst. Practical calculations of the effectiveness factors are presented and earlier results of TAME-synthesis with unsieved Amberlyst 16 as the catalyst $[11,12]$ are recalculated to demonstrate that mass-transfer effects also have to be taken into account in TAME-synthesis, even though the reaction rate is an order of magnitude slower than that of MTBE-synthesis [9].

\section{Experimental}

The effects of temperature and the molar ratio of the reagents on the formation rates of TAME were measured with different Amberlyst 16 particle sizes. The effect of catalyst swelling was studied with several mixtures of reagents ( $\mathrm{MeOH}$ and IA) and product (TAME) on Amberlyst 16.

\subsection{Apparatus}

Reaction rates were measured in a continuous stirred tank reactor (CSTR, $55.6 \mathrm{~cm}^{3}$, stainless steel), where the reaction mixture was magnetically stirred. The stirrer speed was set to $950 \mathrm{rpm}$ to eliminate the influence of external diffusion control on the reaction rates [13]. The catalyst $(0.1856-0.3186 \mathrm{~g}$ dry $)$ was placed in a metal gauze basket ( $60 \mathrm{mesh})$. The temperature $(323-353 \mathrm{~K})$ was controlled within $\pm 0.2 \mathrm{~K}$ by immersing the reactor in a thermostatically controlled water bath. The pressure was kept constant at $0.7 \mathrm{MPa}$ to ensure liquid-phase operation at all temperatures. The pulse-free flow rate $\left(58-65 \mathrm{gh}^{-1}\right)$ of the feed was controlled by a liquid mass flow controller. A Mettler PM 6000 balance was used to measure the actual flow at the outlet of the reactor system. The composition of the feed and the reactor effluent were analysed on-line with a gas chromatograph using an automated liquid sampling valve.

\subsection{Analysis}

Products were analysed with a Hewlett-Packard gas chromatograph 5890 Series II, equipped with a flame ionisation detector using a HP 3396A integrator. The compounds were separated in a glass capillary column HP-1 (length $60 \mathrm{~m}$, thickness $1.0 \mu \mathrm{m}$, column diameter $0.254 \mathrm{~mm}$; Hewlett-Packard). Response factors were determined with calibration solutions.

\subsection{Chemicals and catalyst}

The following reagents were used in the experiments: a mixture of isoamylenes (2M2B $93 \mathrm{wt} . \%$, 2M1B $7 \mathrm{wt} . \%$, Fluka Chemika) and methanol (>99.8 wt.\%, Riedel-de Haën). The ether used for the GC calibration solutions was TAME (>98.5 wt.\%, supplied by Fortum Ltd.). A commercial macroporous cation ion-exchange resin in hydrogen form, Amberlyst 16, was used as the catalyst. The properties of the catalyst are summarised in Table 1. Before the experiments, the catalyst was treated with methanol at room temperature to remove the water from the catalyst structure. The catalyst was stored in methanol.

Table 1

Properties of the studied catalyst A16

\begin{tabular}{lc}
\hline Crosslink level $(\% \mathrm{DVB})$ & 12 \\
Exchange capacity $\left(\mathrm{mmol} \mathrm{g}^{-1}\right)$ & $5.0^{\mathrm{a}}$ \\
Surface area $\left(\mathrm{m}^{2} \mathrm{~g}^{-1}\right)$ & 35 \\
Average pore diameter (̊) & 200 \\
Porosity & 0.25 \\
Particle size (mm) & \\
$\quad$ fraction (I) & $0.50-0.59$ \\
fraction (II) & $0.59-0.71$ \\
$\quad$ fraction (III) & $>0.71$ \\
Exchange capacity (mmol g & \\
$\quad$ fraction (I) & \\
$\quad$ fraction (II) & 4.8 \\
$\quad$ fraction (III) & 4.9 \\
\hline
\end{tabular}

\footnotetext{
${ }^{\mathrm{a}}$ Measured by the manufacturer.
} 


\subsection{Swelling experiments with Amberlyst 16}

The catalyst was dried in an oven $\left(80^{\circ} \mathrm{C}\right)$ and $3 \mathrm{~cm}^{3}$ of dried resin was placed into a byrett. The specific mixture was added into the byrett until the swelling was complete and the colour of the catalyst had changed (from dark brown to light brown). The percentage of swelling at room temperature was calculated according to Eq. (1):

swelling $(\%)=\frac{\text { swollen volume }\left(\mathrm{cm}^{3}\right)}{3 \mathrm{~cm}^{3}} \times 100 \%$

\subsection{Experiments with different A16 particle sizes}

The Amberlyst 16 catalyst was sieved as wet into three fractions, in such a way that the catalyst was immersed in methanol, thus being in the swollen state. The obtained size fractions were (I) $0.50-0.59 \mathrm{~mm}$, (II) $0.59-0.71 \mathrm{~mm}$ and (III) $>0.71 \mathrm{~mm}$ of swollen diameter. We did not grind the resins, although this way the particle size range could have been widened. This was because our aim was to study the size range used industrially. The sulfonic acid content of the fractions were measured by titration [14]. The acid capacity values are presented in Table 1 and as observed, they are quite equal. The reaction rates to TAME were measured with three different molar feed ratios of the reagents (methanol/isoamylene) $(0.3,1.0$ and 3.0) at three different temperatures (323, 343 and $353 \mathrm{~K})$.

During the experiments, the effects of the molar ratio of the reagents were studied in a random sequence, and in each set of different mixtures the temperature was varied randomly. The catalyst was changed after each set of mixtures. The stability of the catalysts was checked by repeating the experiment at the first temperature after the experiments at the other temperatures had been carried out. The catalyst showed no deactivation. Depending on the temperature intervals, it took about $2-4 \mathrm{~h}$ to attain a steady state.

The catalyst weight based reaction rate $r_{\mathrm{obs}}$ $\left(\mathrm{mol} \mathrm{kg}_{\mathrm{cat}}{ }^{-1} \mathrm{~s}^{-1}\right)$ to the product TAME was calculated from the measured amounts of TAME $(\mathrm{g})$ in the product stream according to Eq. (2):

$r_{\mathrm{obs}}=\frac{F_{T, \mathrm{out}}}{W_{\mathrm{cat}}}=\frac{w_{T, \mathrm{out}} \dot{m}_{\mathrm{tot}}}{M_{T} W_{\mathrm{cat}}}$
In order to calculate the characterising parameters of mass-transfer, the reaction rate to TAME $\left(\mathrm{s}^{-1}\right)$ was also calculated by further dividing Eq. (2) with the acid capacity $\left(\mathrm{mol} \mathrm{kg}^{-1}\right)$ of the studied catalyst according to Eq. (3):

$r_{\mathrm{obs}}=\frac{F_{T, \text { out }}}{W_{\mathrm{cat}}\left(\mathrm{H}^{+}\right)}=\frac{w_{T, \text { out }} \dot{m}_{\text {tot }}}{M_{T} W_{\text {cat }}\left(\mathrm{H}^{+}\right)}$

\section{Results and discussion}

\subsection{The effect of resin particle size}

The results of the steady state reaction rates with different methanol/isoamylene feed ratios and with different particle size fractions are presented in Fig. 1a-c. The figures indicate that particle size influences the reaction rate most notably at higher temperatures and when the alkenes are fed in excess, i.e. when the reaction rate should be fast. The influence of particle size on reaction rates is also noticed in stoichiometric conditions and in a less visible way when methanol is fed in excess. The latter is a consequence of the low reaction rates, so that the rates with different particle sizes are almost indistinguishable. Qualitatively, we can therefore conclude that the diffusion rate of the reagents in the pores of the cation-exchange resin has an influence on the observed reaction rate.

\subsection{Calculation of the Weisz-Prater criterion}

The next step was to assess how severe the intraparticle mass-transfer limitations are. In calculation of the well-known Weisz-Prater criterion, we took into account the "true" radius $(R)$ of the particle. The effective radius was calculated from the swelling experiments. These experiments are presented in Table 2.

An empirical equation correlating the amounts (wt.\%) of the components with the swelling (\%) was fitted to these data:

$$
\begin{aligned}
\text { swelling }(\%)= & 1.3 \times w_{\mathrm{MeOH}}+0.7 \times w_{\mathrm{IA}} \\
& +0.7 \times w_{\mathrm{TAME}}
\end{aligned}
$$

where $w_{i}$ are the weight proportions (\%) of the components in the solution. However, it must be pointed out that this equation is a very rough estimate of the 


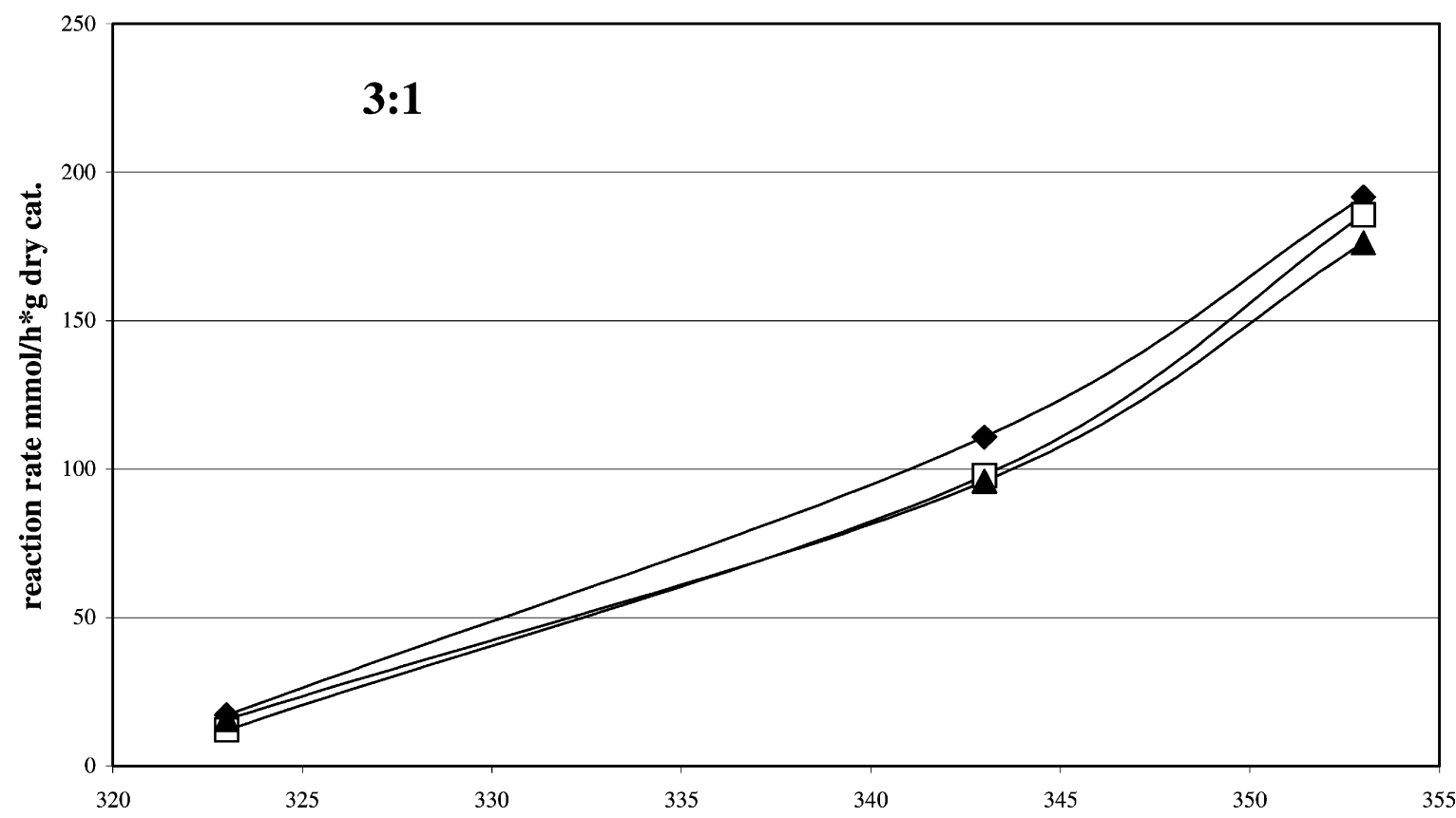

(a)

TEMPERATURE / K

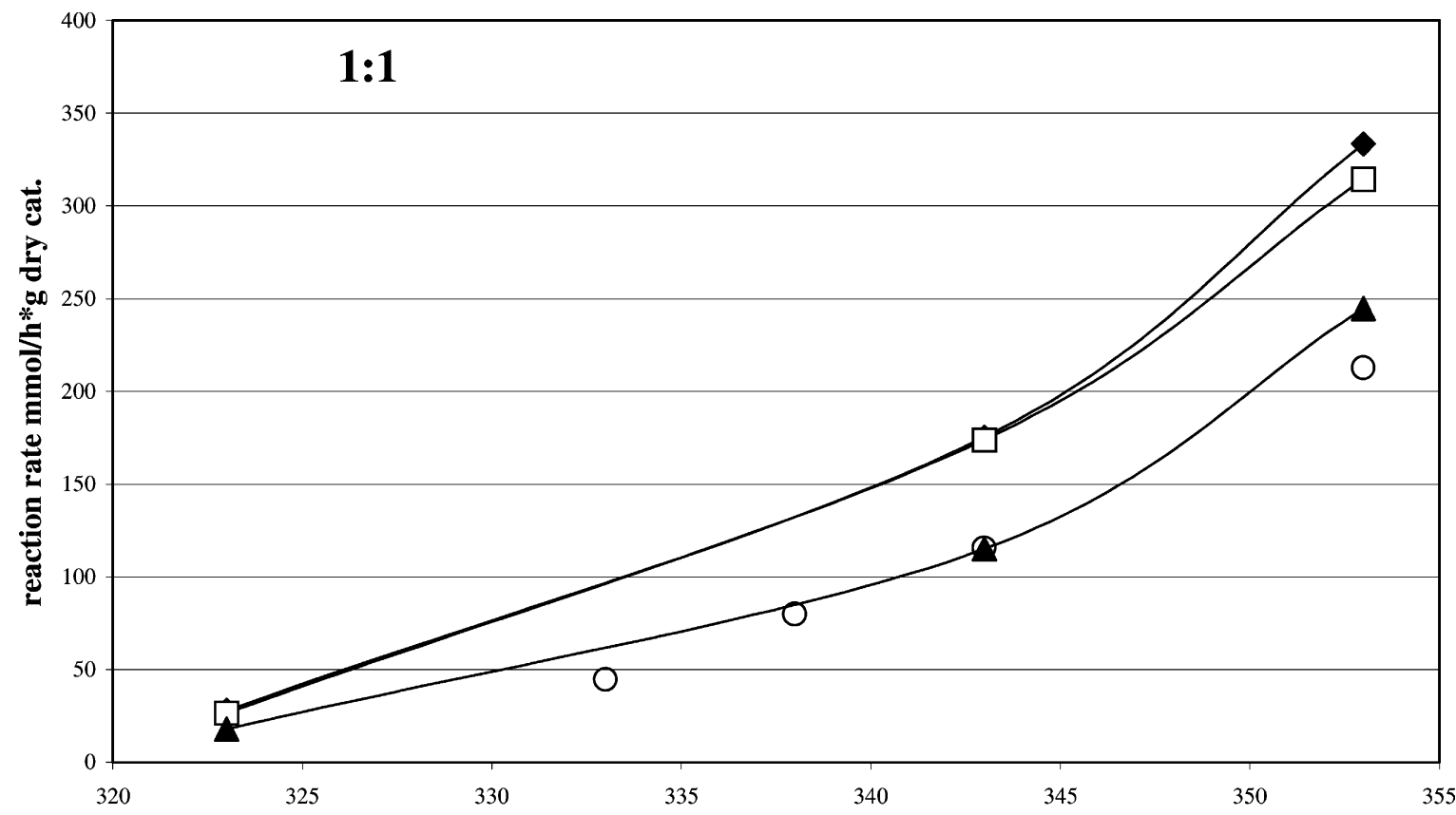

(b)

TEMPERATURE / K

Fig. 1. (a) The effect of temperature on the reaction rates with different particle size fractions of Amberlyst $16((\diamond) 0.50-0.59 \mathrm{~mm}$; $(\square)$ $0.59-0.71 \mathrm{~mm} ;(\mathbf{\Delta})>0.71 \mathrm{~mm})$. Feed ratio of the reagents $\mathrm{MeOH}: \mathrm{IA}=3: 1$. (b) The effect of temperature on the reaction rates with different particle size fractions of Amberlyst $16(\diamond) 0.50-0.59 \mathrm{~mm}$; $(\square) 0.59-0.71 \mathrm{~mm} ;(\boldsymbol{\Delta})>0.71 \mathrm{~mm}$; $(\bigcirc)$ unsieved catalyst. Feed ratio of the reagents $\mathrm{MeOH}: \mathrm{IA}=1: 1$. (c) The effect of temperature on the reaction rates with different particle size fractions $((\bullet) 0.50-0.59 \mathrm{~mm}$; $(\square)$ $0.59-0.71 \mathrm{~mm} ;(\boldsymbol{\Delta})>0.71 \mathrm{~mm})$ of Amberlyst 16. Feed ratio of the reagents MeOH:IA $=1: 3$. 


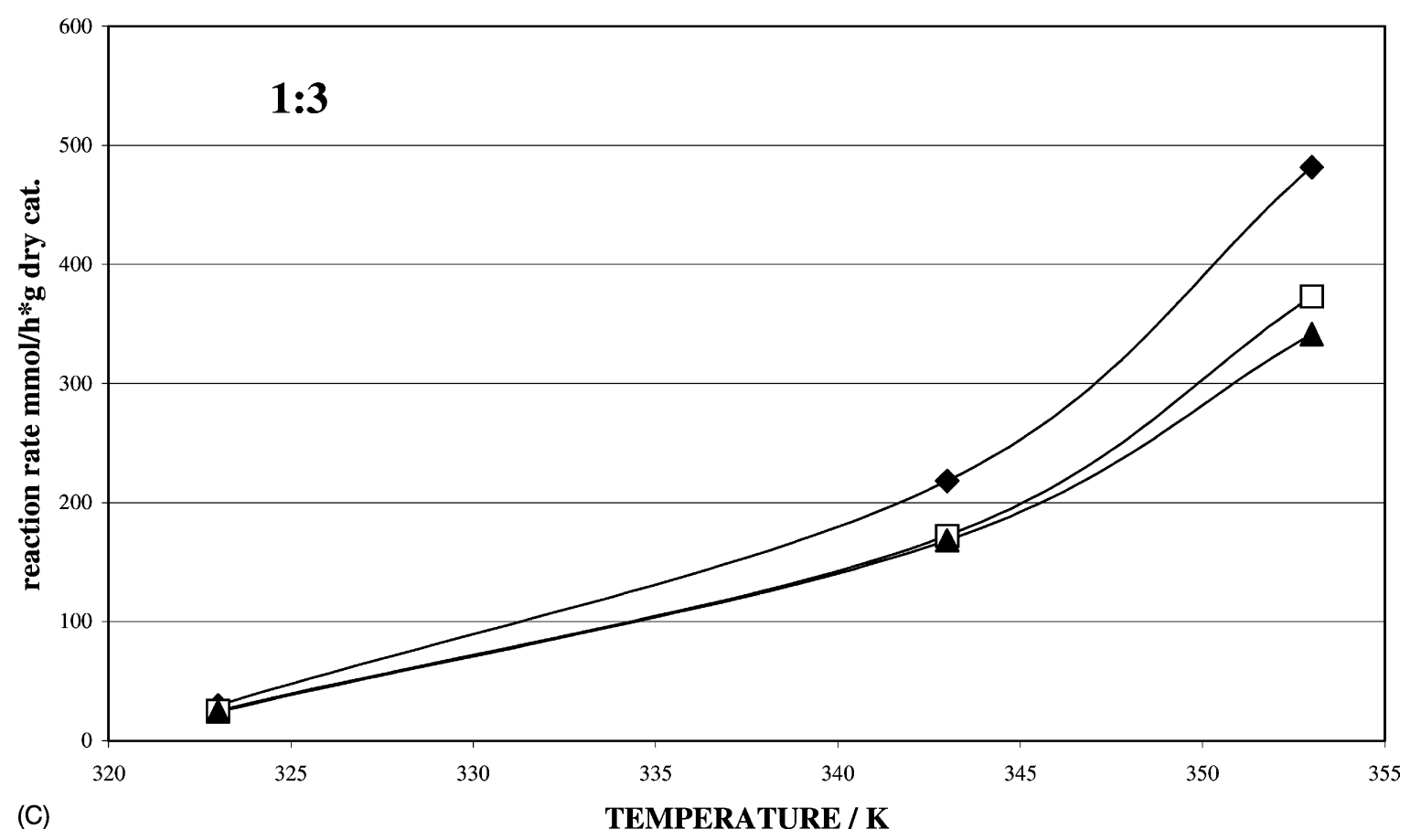

Fig. 1. (Continued).

experiments presented in Table 2. The correlation of the equation with the experimental observations is $0.45275\left(R^{2}\right)$ and six experiments out of nine are predicted more or less satisfactorily with the Eq. (4). This is because the experimental method itself is rather imprecise. Despite this, because there exists no other applicable data either, Eq. (4) will be applied in following Eq. (5).

Table 2

The swelling experiment results with Amberlyst 16

\begin{tabular}{rccc}
\hline MeOH (wt.\%) & IA (wt.\%) & $\begin{array}{l}\text { TAME } \\
(\text { wt.\%) }\end{array}$ & $\begin{array}{l}\text { Swelling (\%) } \\
( \pm 5 \%)\end{array}$ \\
\hline 100 & & & 113 \\
& 100 & & 20 \\
32 & & 100 & 63 \\
82 & 68 & & 120 \\
4 & 18 & & 117 \\
8 & 96 & & 110 \\
75 & 16 & 76 & 83 \\
1 & 4 & 21 & 113 \\
\hline
\end{tabular}

The "true" radius was therefore calculated by

$$
\begin{aligned}
R= & \sqrt[3]{\frac{\text { swelling (\%) by Eq. }(4)}{\text { swelling in pure methanol }(=113 \%)}} \\
& \times \text { mean radius of the fractions I/II } / \text { III }
\end{aligned}
$$

where the mean radius is the mean value of fractions: (I) $0.273 \mathrm{~mm}$; (II) $0.325 \mathrm{~mm}$ and (III) $0.453 \mathrm{~mm}$ (swollen state). We also measured the density of the different particle size fractions by placing catalyst to a byrette filled with methanol and measuring the swollen volume, after which catalyst was dried and weighed. The obtained densities were: (I) 301, (II) 296 and (III) 285 (g dry cat/swollen in methanol $\mathrm{cm}^{3}$ ). The reason for varying densities is attributed to the measuring method. Smaller particles take less space in a byrette and thus the obtained density is greater. For Weisz-Prater criterion the density of a catalyst particle is needed. Volume of a cube whose side equals the mean diameter of a particle $0.545 \mathrm{~mm}$ (fraction I) is 1.9 times the volume of sphere whose mean radius is of $0.273 \mathrm{~mm}$. Based on the results presented 
above, $\rho_{\mathrm{p}}$ can be approximated to be $2 \times 300=$ $600 \mathrm{~kg} \mathrm{dm}^{-3}$.

For the Weisz-Prater criterion an estimate of the effective diffusion coefficient is needed. We chose the Scheibel-method [15] corrected to liquid-phase activities with the Vignes-equation [16] to calculate the bulk liquid diffusion coefficients. The accuracy of the Scheibel-method was found to be $\pm 20 \%$ in the study of $\mathrm{Li}$ and Carr [17] and they recommend its use over the Wilke-Chang-method if measured diffusion coefficients are not available, as in our case. The Scheibel equation for infinite dilution is:

$D_{A, B}^{0}\left(\mathrm{~cm}^{2} / \mathrm{s}\right)=\frac{8.2 \times 10^{-8} T}{\mu_{\mathrm{B}} V_{\mathrm{A}}^{1 / 3}}\left[1+\left(\frac{3 V_{\mathrm{B}}}{V_{\mathrm{A}}}\right)^{2 / 3}\right]$

where A and B denote the solute and solvent, respectively, $V$ the molar volume $\left(\mathrm{cm}^{3} \mathrm{~mol}^{-1}\right), \mu$ the viscosity (cP) and $T$ the temperature (K). The molar volumes and the viscosities of the components (at temperature $298.15 \mathrm{~K}$ ) are presented in Table 3.

The viscosities were extrapolated to the studied temperatures with the equation of Reid et al. [21]:

$\mu_{\mathrm{L}}^{-0.2661}=\mu_{\mathrm{K}}^{-0.2661}+\frac{T-T_{\mathrm{K}}}{233}$

where $\mu_{\mathrm{K}}$ is a known value at $T_{\mathrm{K}}$.

As already mentioned, the infinite dilution diffusion coefficients were corrected to liquid-phase activities according to the Vignes equation as proposed by Rehfinger and Hoffmann [22] for MTBE:

$D_{\mathrm{A}, \mathrm{B}}=\left(\frac{D_{\mathrm{B}, \mathrm{A}}^{0}}{D_{\mathrm{A}, \mathrm{B}}^{0}}\right)^{X}\left(\frac{\ln a}{\ln x}\right) D_{\mathrm{A}, \mathrm{B}}^{0}$

The liquid-phase activities were calculated with the formerly determined binary interaction parameters of the Wilson method [23]. The Wilson method

Table 3

Molar volumes and viscosities of the components

\begin{tabular}{llll}
\hline & $\begin{array}{l}\text { Molar volume } \\
\left(\mathrm{cm}^{3} \mathrm{~mol}^{-1}\right)\end{array}$ & $\begin{array}{l}\text { Viscosity } \\
\left(\mathrm{mPa} \mathrm{s} \text { at } 25^{\circ} \mathrm{C}\right)\end{array}$ & Reference \\
\hline $\mathrm{MeOH}$ & 40.73 & 0.553 & {$[18]$} \\
2M1B & 107.8 & 0.203 & Assumed (2M2B) \\
2M2B & 105.9 & 0.203 & {$[19]$} \\
TAME & 133.5 & 0.438 & {$[20]$} \\
\hline
\end{tabular}

was chosen because it is particularly suitable for alcohol-hydrocarbon mixtures [21]. Because the studied reaction forms a ternary system $(\mathrm{MeOH}$, isoamylene, TAME), the equation by Kooijman and Taylor [24] was further applied to estimate the diffusion coefficients in the multicomponent mixture:

$$
D_{i, j}=\left(D_{i j}^{0}\right)^{X_{j}}\left(D_{j i}^{0}\right)^{X_{i}} \prod_{\substack{k=1 \\ k \neq i, j}}^{n}\left(D_{i k}^{0} D_{j k}^{0}\right)^{X_{k} / 2}
$$

In this way, we obtained the isoamylene bulk diffusion coefficients in methanol and TAME, or vice-versa the methanol bulk diffusion coefficients in isoamylene and TAME. They were further converted to effective diffusion coefficients by assuming the tortuosity $(\tau)$ to be 4 as recommended by Satterfield [25] for ion-exchange resins and knowing the dry state porosity $(\varepsilon)$ of Amberlyst 16 to be of 0.25 (measured by the manufacturer) so that the swollen state porosity could be assumed to be about double $=0.5$ (on basis of the swelling experiments, Table 2):

$D_{\mathrm{e}, \mathrm{IA}}=D_{\mathrm{IA}, M, T} \frac{\varepsilon}{\tau}$
$D_{\mathrm{e}, M}=D_{M, \mathrm{IA}, T} \frac{\varepsilon}{\tau}$

The isoamylene and methanol concentrations were calculated from the measured amounts at the outlet $\left(\mathrm{molh}^{-1}\right)$ and the estimated densities of the whole mixtures $\left(\mathrm{kg} \mathrm{m}^{-3}\right)$. The liquid densities of the product streams were calculated with FLOWBAT by applying the model of Aalto et al. [26]. Although the model was originally developed for hydrocarbon mixtures, the authors tested it against liquid density data for alcohol-hydrocarbon mixtures. The average absolute deviation was found to be less than $5 \%$, a level of accuracy adequate for our purposes since the concentrations are used only for the Weisz-Prater criterion, which is a very rough estimate. This conventional textbook method to unconventional catalyst has also been applied by Yadav and Thathagar [27] in esterification of maleic acid with ethanol over cation-exchange resin catalysts.

Finally the Weisz-Prater criterion,

$\Phi=\frac{-r_{\mathrm{obs}} \rho_{\mathrm{p}} R^{2}}{D_{\mathrm{e}, \mathrm{IA}} C_{\mathrm{IA}}}$ 


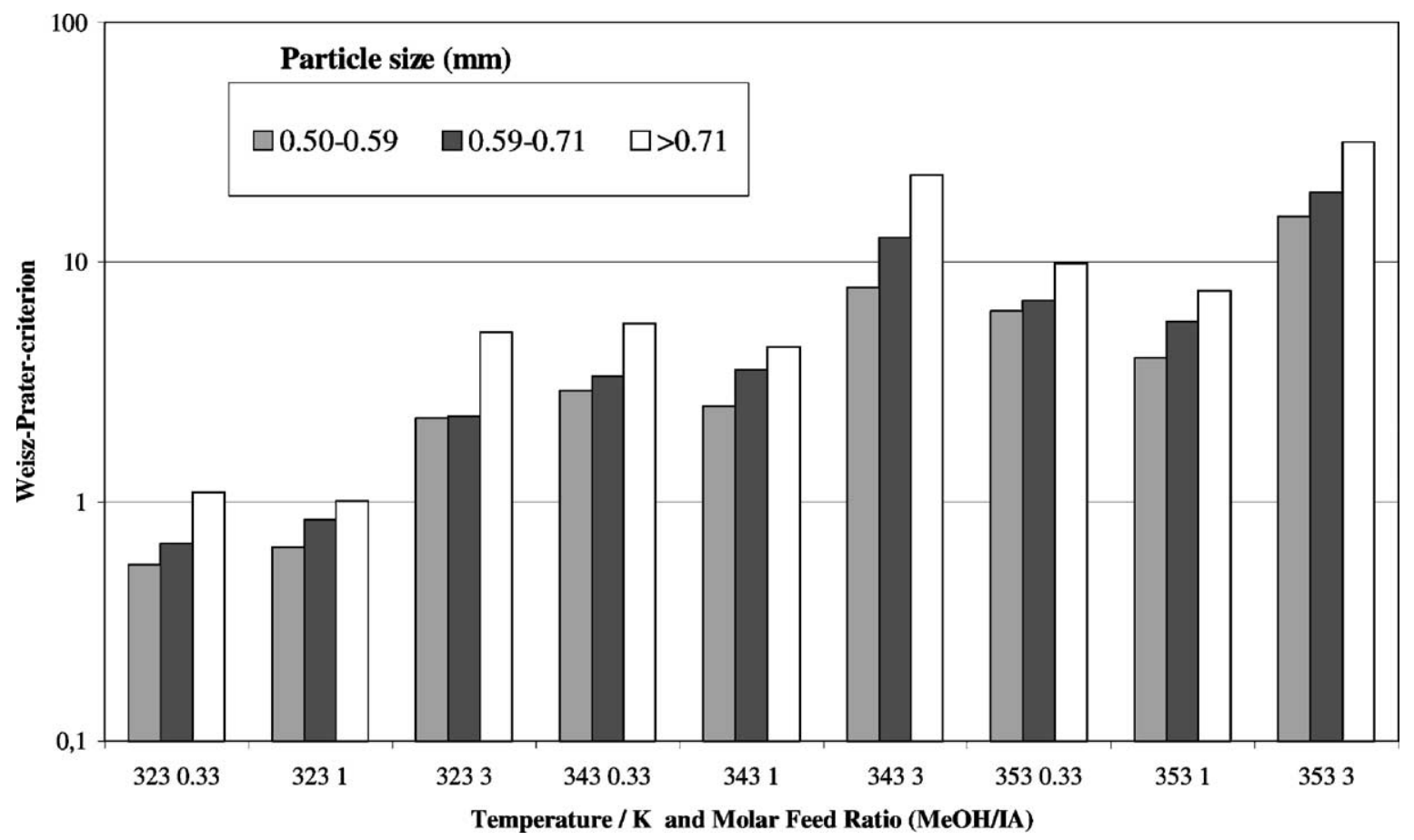

Fig. 2. The calculated Weisz-Prater criterion under different experimental conditions.

$\Phi=\frac{-r_{\mathrm{obs}} \rho_{\mathrm{p}} R^{2}}{D_{\mathrm{e}, M} C_{M}}$

states that diffusion does not limit the reaction if the value of Eq. (11) is much less than 1. Fig. 2 shows the results of our calculations under different experimental conditions. The figure indicates that the mass-transfer severely limits the reaction rate at higher temperatures $(\geq 343 \mathrm{~K})$ and under conditions of high methanol concentration. The calculations are presented in Table 4.

\subsection{Calculation of the effectiveness factors}

The second step was to calculate the Thiele modulus for the experimental runs. In an earlier study [12], we found out that a Langmuir-Hinshelwood type model described our rate data from batch reactor experiments best:

$r\left(s^{-1}\right)=\frac{k_{1}\left(K_{1 b} / K_{M}\right) a_{M} a_{1 b}\left(1-a_{T} / K_{1} a_{M} a_{1 b}\right)+k_{3}\left(K_{2 b} / K_{M}\right) a_{M} a_{2 b}\left(1-a_{T} / K_{2} a_{M} a_{2 b}\right)}{\left(K_{T} / K_{M} a_{T}+a_{M}+K_{1 b} / K_{M} a_{1 b}+K_{2 b} / K_{M} a_{2 b}\right)^{2}}$

The Thiele modulus can be calculated from the correlation by Aris [28] for a second-order LangmuirHinshelwood type kinetics:

$\phi=\frac{R}{3} \frac{\sqrt{\begin{array}{c}\left(r_{T}\right)^{s}\left(K_{M} /\left(D_{\mathrm{e}, M}\right)\right. \\ \left.+K_{\mathrm{IA}} /\left(D_{\mathrm{e}, \mathrm{IA}}\right)-K_{T} /\left(D_{\mathrm{e}, T}\right)\right)\end{array}}}{M}$

where,

$$
\begin{aligned}
M= & (1+\kappa)\left[\frac { 2 } { 1 + \varepsilon _ { 0 } } \left\{\left(\varepsilon_{0}-2 \kappa\right) \ln \frac{1+\kappa}{\kappa}\right.\right. \\
& \left.\left.+\frac{1+2 \kappa-\varepsilon_{0}}{1+\kappa}\right\}\right]^{1 / 2}
\end{aligned}
$$

and

$\kappa=K_{M} a_{M}^{s}+K_{\mathrm{IA}} a_{\mathrm{IA}}^{s}+K_{T} a_{T}^{s}$ 
Table 4

Calculations of the Weisz-criterion and the effectiveness factors

\begin{tabular}{|c|c|c|c|c|c|c|c|c|}
\hline Fraction number & Feed $\mathrm{MeOH} / \mathrm{IA}$ & Swelling (\%) & $R(\mathrm{~cm})$ & Rate $\left(\mathrm{mol} /(\mathrm{kg} \text { cat s})^{-1}\right)$ & WEISZ & $k_{3}(1 / \mathrm{s})$ & $\phi$ & $\eta$ \\
\hline \multicolumn{9}{|l|}{$323 \mathrm{~K}$} \\
\hline \multirow[t]{3}{*}{ I } & $1 / 1$ & 86 & 0.0249 & 0.0077 & 0.64 & 0.046 & 0.4 & 0.95 \\
\hline & $3 / 1$ & 102 & 0.0263 & 0.0048 & 2.22 & 0.033 & 0.6 & 0.85 \\
\hline & $1 / 3$ & 75 & 0.0238 & 0.0082 & 0.54 & 0.045 & 0.2 & 1.00 \\
\hline \multirow[t]{4}{*}{ II } & $1 / 1$ & 85 & 0.0295 & 0.0074 & 0.84 & 0.043 & 0.4 & 0.95 \\
\hline & $3 / 1$ & 102 & 0.0314 & 0.0034 & 2.27 & 0.023 & 0.6 & 0.85 \\
\hline & $3 / 1$ & 102 & 0.0314 & 0.0034 & 2.29 & 0.023 & 0.6 & 0.85 \\
\hline & $1 / 3$ & 74 & 0.0283 & 0.0070 & 0.67 & 0.038 & 0.2 & 1.00 \\
\hline \multirow[t]{3}{*}{ III } & $1 / 1$ & 85 & 0.0389 & 0.0049 & 1.00 & 0.029 & 0.5 & 0.90 \\
\hline & $3 / 1$ & 102 & 0.0413 & 0.0044 & 5.09 & 0.030 & 0.8 & 0.78 \\
\hline & $1 / 3$ & 75 & 0.0374 & 0.0068 & 1.09 & 0.037 & 0.3 & 0.98 \\
\hline \multicolumn{9}{|l|}{$343 \mathrm{~K}$} \\
\hline \multirow[t]{4}{*}{ I } & $1 / 1$ & 84 & 0.0246 & 0.0487 & 2.50 & 0.440 & 0.7 & 0.90 \\
\hline & $3 / 1$ & 101 & 0.0263 & 0.0308 & 10.29 & 0.335 & 1.1 & 0.71 \\
\hline & $3 / 1$ & 101 & 0.0263 & 0.0242 & 7.85 & 0.255 & 1.0 & 0.73 \\
\hline & $1 / 3$ & 73 & 0.0236 & 0.0606 & 2.90 & 0.487 & 0.4 & 0.95 \\
\hline \multirow[t]{5}{*}{ II } & $1 / 1$ & 84 & 0.0294 & 0.0483 & 3.56 & 0.440 & 0.8 & 0.78 \\
\hline & $1 / 1$ & 85 & 0.0295 & 0.0457 & 3.46 & 0.414 & 0.8 & 0.78 \\
\hline & $3 / 1$ & 101 & 0.0314 & 0.0272 & 12.65 & 0.283 & 1.3 & 0.67 \\
\hline & $1 / 3$ & 73 & 0.0281 & 0.0479 & 3.32 & 0.377 & 0.4 & 0.95 \\
\hline & $1 / 3$ & 73 & 0.0282 & 0.0482 & 3.30 & 0.384 & 0.4 & 0.95 \\
\hline \multirow[t]{4}{*}{ III } & $1 / 1$ & 85 & 0.0388 & 0.0320 & 4.29 & 0.279 & 0.9 & 0.76 \\
\hline & $1 / 1$ & 85 & 0.0388 & 0.0325 & 4.41 & 0.281 & 1.0 & 0.73 \\
\hline & $3 / 1$ & 102 & 0.0413 & 0.0266 & 23.09 & 0.284 & 1.7 & 0.55 \\
\hline & $1 / 3$ & 73 & 0.0370 & 0.0468 & 5.55 & 0.370 & 0.6 & 0.85 \\
\hline \multicolumn{9}{|l|}{$353 \mathrm{~K}$} \\
\hline \multirow[t]{4}{*}{ I } & $1 / 1$ & 83 & 0.0246 & 0.0926 & 3.98 & 1.108 & 0.8 & 0.80 \\
\hline & $3 / 1$ & 101 & 0.0262 & 0.0532 & 15.46 & 0.751 & 1.3 & 0.67 \\
\hline & $1 / 3$ & 71 & 0.0234 & 0.1338 & 6.22 & 1.358 & 0.5 & 0.90 \\
\hline & $1 / 3$ & 72 & 0.0234 & 0.1266 & 5.77 & 1.278 & 0.5 & 0.90 \\
\hline \multirow[t]{3}{*}{ II } & $1 / 1$ & 84 & 0.0294 & 0.0873 & 5.62 & 1.043 & 1.0 & 0.73 \\
\hline & $3 / 1$ & 101 & 0.0313 & 0.0502 & 19.61 & 0.676 & 1.5 & 0.60 \\
\hline & $1 / 3$ & 71 & 0.0279 & 0.1036 & 6.90 & 0.973 & 0.5 & 0.90 \\
\hline \multirow[t]{4}{*}{ III } & $1 / 1$ & 84 & 0.0387 & 0.0680 & 7.58 & 0.724 & 1.2 & 0.70 \\
\hline & $3 / 1$ & 100 & 0.0411 & 0.0630 & 40.92 & 0.836 & 2.2 & 0.48 \\
\hline & $3 / 1$ & 100 & 0.0411 & 0.0491 & 31.58 & 0.627 & 2.0 & 0.50 \\
\hline & $1 / 3$ & 73 & 0.0369 & 0.0948 & 9.89 & 0.928 & 0.7 & 0.82 \\
\hline
\end{tabular}

and

$\varepsilon_{0}=\frac{\left(D_{\mathrm{e}, \mathrm{IA}}\right) a_{\mathrm{IA}}^{s}}{\left(D_{\mathrm{e}, M}\right) a_{M}^{s}}-1$

The activities of the components were calculated with the Wilson method [23]. The equilibrium compositions have been published by Rihko-Struckmann et al. [23]. The temperature dependencies of the equilibrium constants are presented in Eqs. (17) and (18):

$K_{1}=\exp \left(-8.74435+\frac{4142.069}{T}\right)$

$$
K_{2}=\exp \left(-8.24371+\frac{3219.118}{T}\right)
$$

The relative adsorption equilibrium constants were calculated from the study by Oktar et al. [29]:

$\frac{K_{1 b}}{K_{M}}=\exp \left(-13.0304+\frac{3171.451}{T}\right)$

$$
\frac{K_{2 b}}{K_{M}}=\exp \left(-9.22212+\frac{1852.525}{T}\right)
$$




$$
\frac{K_{T}}{K_{M}}=\exp \left(-7.32796+\frac{1050.648}{T}\right)
$$

The effective radius $(R)$ and the effective diffusion coefficients were calculated as previously (Eqs. (5), (6)-(10), respectively). Knowing the Thiele modulus we could further estimate the magnitude of the effectiveness factor $(\eta)$ of the experimental runs from the generalised graphs of Aris [28].

The graphically estimated values of the effectiveness factor are presented in Table 4 . The value of the effectiveness factor varies between 0.5 and 1 . The determined effectiveness factors for rate parameter $k_{3}$ are presented in Fig. 3. The effectiveness factor decreases with increasing particle size and increasing temperature. The decreasing effects of temperature and particle size are more significant with excess alcohol in the feed.

\subsection{Combining the reaction and the mass-transfer}

In the earlier study [11], we presented the results of the batch reactor experiments where the effect of temperature $(333-353 \mathrm{~K})$ and reagents feed molar ratio $(\mathrm{MeOH} / \mathrm{IA}=0.2-2.0)$ on the synthesis of TAME were measured with Amberlyst 16 as catalyst. A kinetic model of Eley-Rideal type was proposed in that paper. Later on we carried out additional experiments at $333 \mathrm{~K}$ with pure $2 \mathrm{M} 1 \mathrm{~B}$ as reagent, varying the reagents initial molar ratio $(\mathrm{MeOH} / 2 \mathrm{M} 1 \mathrm{~B}=$ $0.2-4.0)$. The aim was to study the isomerisation reaction more deeply and further kinetic modelling was also carried out. A revised kinetic model of Langmuir-Hinshelwood type was presented in that later paper [12]. However, because unsieved catalyst had been used in most of those experiments, we could not be sure whether our kinetic results were truly

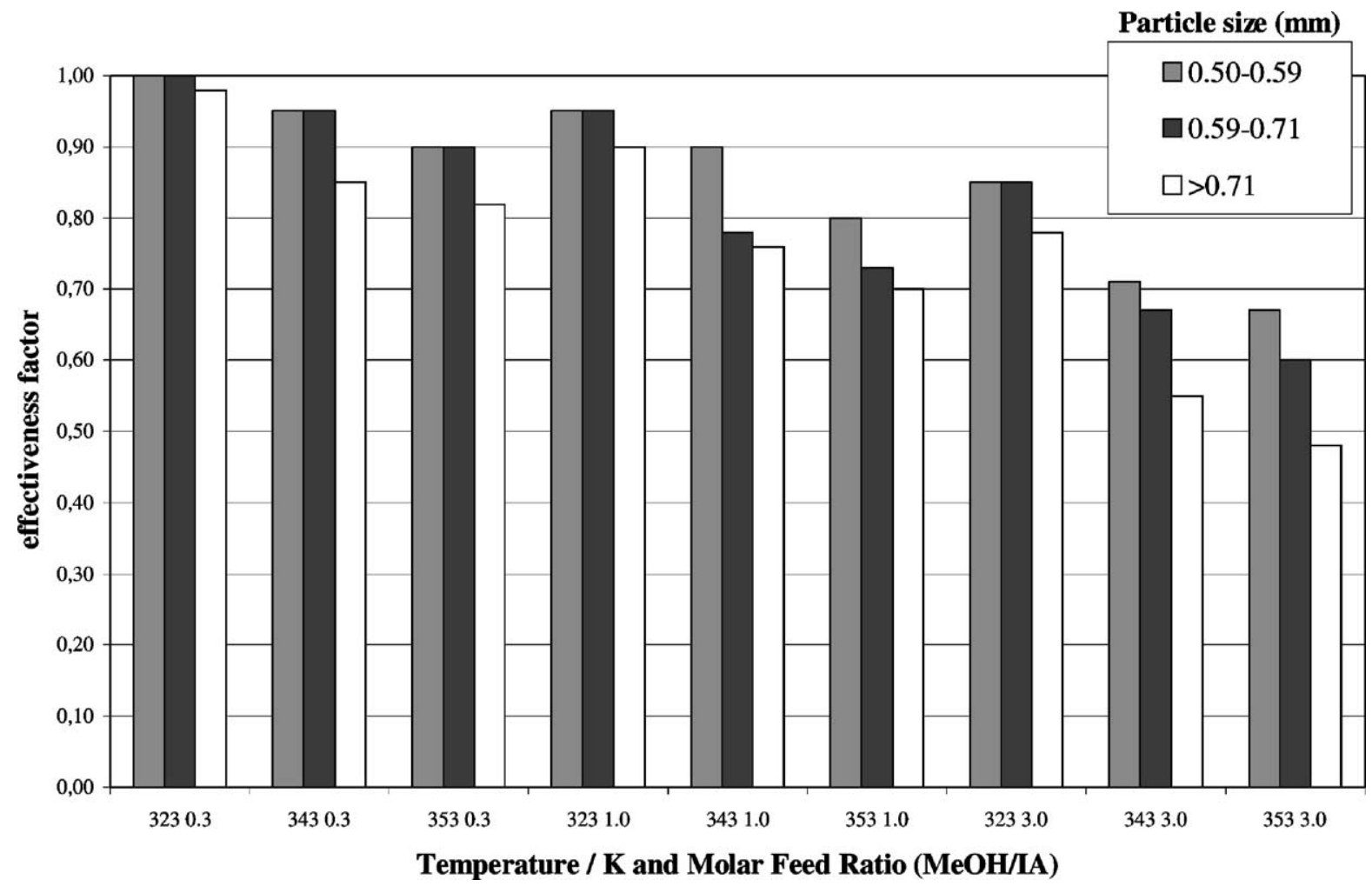

Fig. 3. The calculated effectiveness factor of rate parameter $k_{3}$ under different experimental conditions. 


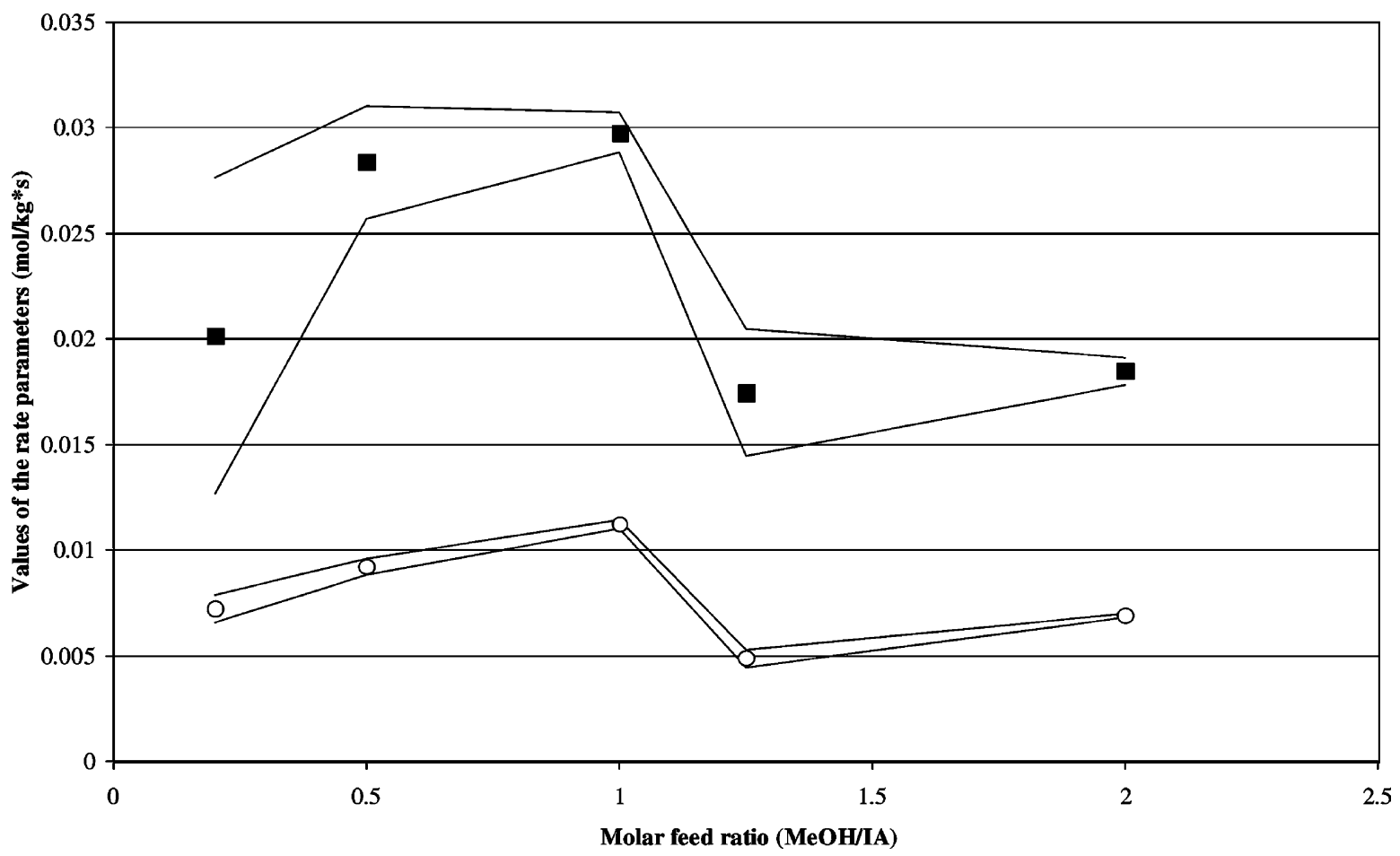

Fig. 4. The estimated values of the rate parameters $k_{1}(\boldsymbol{\square})$ and $k_{3}(\bigcirc)$ and their confidence limits $(---= \pm$ standard error) at $333 \mathrm{~K}$ as a function of reagents (MeOH/IA) initial molar ratio (for experimental, see [11]).

"intrinsic" or not. We found out that the values of the rate parameters were strongly dependent on the reagents initial molar ratio. In Fig. 4, the values of the etherification rate parameters obtained with the basic Langmuir-Hinshelwood type model, assuming that the adsorption of alcohol is dominant (Eq. (22)), are presented as a function of feed molar ratio:

$$
\begin{aligned}
r\left(s^{-1}\right)= & \frac{k_{1}^{\prime} a_{1 b}}{a_{M}}\left(1-\frac{a_{T}}{a_{1 b} a_{M} K_{1}}\right) \\
& +\frac{k_{3}^{\prime} a_{2 b}}{a_{M}}\left(1-\frac{a_{T}}{a_{2 b} a_{M} K_{2}}\right)
\end{aligned}
$$

Even though the nonideality of the system has been taken into account by calculating the liquid-phase activities of the components with the Wilson method, the rate parameter values are not constant. The confidence values of the parameters are presented in Fig. 4 and neither they explain the variations. Explanation could be mass-transfer effects as shown earlier in this paper.
It is possible to make a simple recalculation from those batch reactor results $[11,12]$. The measured rates with unsieved catalyst correspond to measured rates with fraction III of this study, e.g. see Fig. 1b, where the measured reaction rates with unsieved catalyst under stoichiometric conditions (circles) are presented. The effectiveness factors of those earlier batch reactor experiments $[11,12]$ under different intermediate experimental conditions (feed molar ratio (0.5-2.0) and temperature (333-353 K)) can be interpolated from the results presented in Table 4 . When modifying the rate constants obtained from the batch reactor experiments $[11,12]$ by dividing them by the effectiveness factors estimated in this study, the $\mathrm{R}$-squared values of the regression analysis against temperature increase (better fit for Arrhenius-type dependency). The activation energies increase by about $9 \mathrm{~kJ} \mathrm{~mol}^{-1}$ to $82 \mathrm{~kJ} \mathrm{~mol}^{-1}$ for the etherification of $2 \mathrm{M} 1 \mathrm{~B}$ and to $95 \mathrm{~kJ} \mathrm{~mol}^{-1}$ for the etherification of $2 \mathrm{M} 2 \mathrm{~B}$. The results of the calculations are presented in Table 5 . 
Table 5

Recalculation of earlier results $[11,12]$

\begin{tabular}{|c|c|c|c|c|c|c|}
\hline Feed MeOH/IA & Temperature $(\mathrm{K})$ & $k_{1}$ & $k_{3}$ & $\eta$ & $k_{1} / \eta$ & $k_{3} / \eta$ \\
\hline 2 & 333.15 & 0.019 & 0.007 & 0.75 & 0.025 & 0.009 \\
\hline 1 & 333.15 & 0.030 & 0.011 & 0.83 & 0.036 & 0.014 \\
\hline 0.5 & 333.15 & 0.028 & 0.009 & 0.89 & 0.032 & 0.010 \\
\hline 2 & 338.15 & 0.027 & 0.011 & 0.70 & 0.039 & 0.016 \\
\hline 1 & 338.15 & 0.044 & 0.020 & 0.80 & 0.055 & 0.025 \\
\hline 0.5 & 338.15 & 0.046 & 0.021 & 0.86 & 0.053 & 0.025 \\
\hline 2 & 343.15 & 0.044 & 0.019 & 0.66 & 0.067 & 0.029 \\
\hline 1 & 343.15 & 0.070 & 0.032 & 0.76 & 0.092 & 0.042 \\
\hline 0.5 & 343.15 & 0.063 & 0.018 & 0.82 & 0.076 & 0.022 \\
\hline 2 & 353.15 & 0.098 & 0.041 & 0.59 & 0.166 & 0.070 \\
\hline 1 & 353.15 & 0.105 & 0.061 & 0.70 & 0.151 & 0.088 \\
\hline \multirow[t]{3}{*}{0.5} & 353.15 & 0.132 & 0.068 & 0.79 & 0.168 & 0.087 \\
\hline & & $0.885^{\mathrm{a}}$ & $0.872^{\mathrm{a}}$ & & $0.956^{\mathrm{a}}$ & $0.926^{\mathrm{a}}$ \\
\hline & & $72800^{\mathrm{b}}$ & $86700^{\mathrm{b}}$ & & $81500^{\mathrm{b}}$ & $95400^{\mathrm{b}}$ \\
\hline
\end{tabular}

${ }^{\text {a }}$ Regression values $\left(R^{2}\right)$.

${ }^{\mathrm{b}}$ Activation energy, $E_{\text {act }}\left(\mathrm{J} \mathrm{mol}^{-1}\right)$.

The above treatment shows that intraparticle diffusion influences the reaction rate in TAME-synthesis, and that a significant improvement for the correlation between the kinetic model and the experiments can be obtained, if effectiveness factors are taken into account.

\subsection{Comparison of activation energies}

The activation energies of rate constant $k_{3}$ (Eq. (12)) of the kinetic experiments with the different particle sizes and feed conditions presented in this study are summarised in Table 6 . Table 6 shows that the activation energies with different particle size fractions $\left(100-108 \mathrm{~kJ} \mathrm{~mol}^{-1}\right)$ calculated with the more precise model (Eq. (12)) are higher than the value $\left(95 \mathrm{~kJ} \mathrm{~mol}^{-1}\right)$ obtained from the recalculated batch reactor experiments [11,12] with the basic model (Eq. (22)). The difference is attributed to the differ-

Table 6

Activation energies with different catalyst fractions

\begin{tabular}{llll}
\hline Feed ratio MeOH:IA & \multicolumn{3}{l}{ Activation energies (kJ mol) } \\
\cline { 2 - 4 } & I & II & III \\
\hline $1: 1$ & 101 & 102 & 102 \\
$3: 1$ & 101 & 108 & 105 \\
$1: 3$ & 108 & 103 & 102 \\
\hline
\end{tabular}

ence between the models. In Eq. (22), the adsorption equilibrium constants are lumped with the rate coefficients $k_{1}^{\prime}$ and $k_{3}^{\prime}$ and this results in lower apparent activation energies.

The comparison of activation energies (Table 6) does not indicate strong diffusion limitations. In a recent study by Jin et al. [30] the kinetics of the synthesis of TAME in an internal recycle gradient-less reactor with an ion-exchange resin NKC-9 as a catalyst has been studied. The authors found that the Langmuir-Hinshelwood type model was particularly appropriate and derived activation energies of $96.78 \mathrm{~kJ} \mathrm{~mol}^{-1}$ for the etherification of $2 \mathrm{M} 1 \mathrm{~B}$ and $102.4 \mathrm{~kJ} \mathrm{~mol}^{-1}$ for the etherification of $2 \mathrm{M} 2 \mathrm{~B}$. Our activation energies $\left(100-108 \mathrm{~kJ} \mathrm{~mol}^{-1}\right)$ presented in Table 6 are thus in satisfactory agreement with the latter value. However, in conditions of excess alkenes in the feed $(\mathrm{MeOH}: \mathrm{IA}=1: 3)$ the activation energy is lower with larger particles of A16. This is because the reaction rate is faster, so that it becomes diffusion limited in a more visible way. Under other conditions, a similar trend cannot be observed: the activation energy varies randomly due to experimental error.

\section{Conclusions}

The mass-transfer of the reacting components inside the pores of the cationic ion-exchange resin Amberlyst 
16 was estimated from experiments with different resin particle sizes. The measured steady state reaction rates decreased with increasing particle size most notably at higher temperatures and with substoichiometric feed of the reagents (methanol/isoamylenes), i.e. when the reaction rate should be fast. The estimated effectiveness factors decreased with increasing particle size and increasing temperature. The decreasing effects of temperature and particle size on the effectiveness factors under nonstoichiometric conditions were more significant with excess alcohol in the feed. The batch reactor results of the previous studies were recalculated by dividing the rate constants of the TAME-synthesis by the effectiveness factors obtained from the present study. The recalculations resulted in a better fit and the obtained activation energies increased by about $9 \mathrm{~kJ} \mathrm{~mol}^{-1}$, thus being in a more acceptable range of intrinsic values.

\section{Acknowledgements}

The financial support of the Academy of Finland through the Graduate School in Chemical Engineering (GSCE) for P.K.P is highly appreciated. Jani Porkka, Riikka Puurunen, and Jaakko Ruokomäki are thanked for the experimental assistance.

\section{References}

[1] J. Tejero, M. Cunill, M. Iborra, J. Mol. Catal. 42 (1987) 257.

[2] B. Corain, M. Zecca, K. Jerábek, J. Mol. Catal. 177 (2001) 3.

[3] V.P. Gupta, W.J.M. Douglas, AIChE J. 13 (1967) 883.

[4] E. Velo, L. Puigjaner, F. Recasens, Ind. Eng. Chem. Res. 29 (1990) 1485.

[5] S.-K. Ihm, M.-J. Chung, K.-Y. Park, Ind. Eng. Chem. Res. 27 (1988) 41.
[6] D.A. Berg, T.J. Harris, Ind. Eng. Chem. Res. 32 (1993) 2147.

[7] K. Sundmacher, U. Hoffmann, Chem. Eng. Sci. 49 (1994) 3077.

[8] F.H. Syed, R. Datta, Ind. Eng. Chem. Res. 41 (2002) 1754.

[9] A. Higler, R. Krishna, R. Taylor, Ind. Eng. Chem. Res. 39 (2000) 1596.

[10] R. Pla, J. Tejero, F. Cunill, J.F. Izquierdo, M. Iborra, C. Fité, Stud. Surf. Sci. Catal. 130 (2000) 2609.

[11] L.K. Rihko, P.K. Kiviranta-Pääkkönen, A.O.I. Krause, Ind. Eng. Chem. Res. 36 (1997) 614.

[12] P. Kiviranta-Pääkkönen, A.O.I. Krause, Chem. Eng. Technol., in press.

[13] L.K. Rihko, A.O.I. Krause, Ind. Eng. Chem. Res. 34 (1995) 1172.

[14] ASTM D 2187, 1991.

[15] E.G. Scheibel, Ind. Eng. Chem. 46 (1954) 2007.

[16] A. Vignes, I\&EC Fundamentals 5 (1966) 189.

[17] J. Li, P. Carr, Anal. Chem. 69 (1997) 2530.

[18] TRC Thermodynamic Tables, Thermodynamic Research Center, Texas A\&M University, College Station, TX, 1998.

[19] CRC Handbook of Chemistry and Physics, 63rd ed., CRC Press, USA, 1984.

[20] A. Pal, G. Dass, J. Chem. Eng. Data 44 (1999) 1325.

[21] R.C. Reid, J.M. Prausnitz, B.E. Poling, The Properties of Gases and Liquids, fourth ed., McGraw-Hill, New York, 1988, p. 439.

[22] A. Rehfinger, U. Hoffmann, Chem. Eng. Sci. 45 (1990) 1619.

[23] L.K. Rihko-Struckmann, J.A. Linnekoski, O.S. Pavlov, A.O.I. Krause, J. Chem. Eng. Data 45 (2000) 1030.

[24] H. Kooijman, R. Taylor, Ind. Eng. Chem. Res. 30 (1991) 1217.

[25] C. Satterfield, Mass Transfer in Heterogeneous Catalysis, MIT Press, Massachusetts, 1970.

[26] M. Aalto, K.I. Keskinen, J. Aittamaa, S. Liukkonen, Fluid Phase Equilib. 114 (1996) 21.

[27] G.D. Yadav, M.B. Thathagar, React. Funct. Polym. 52 (2002) 99.

[28] R. Aris, The Mathematical Theory of Diffusion and Reaction in Permeable Catalysts, vol. I, Clarendon Press, Oxford, 1975, p. 168.

[29] N. Oktar, K. Mürtezaogly, T. Dogu, G. Dogu, Can. J. Chem. Eng. 77 (1999) 406.

[30] H.-B. Jin, F.-R. Xiao, C.-Y. Yang, Z.-M. Tong, Shiyou Huagong Gaodeng Xuexiao Xuebao 15 (2002) 26. 